\title{
Adaptive Feed-forward Automatic Gauge Control in Hot Strip Finishing Mill
}

\author{
Young Kow LEE, ${ }^{1)}$ Yu Jin JANG ${ }^{21}$ and Sang Woo KIM ${ }^{11}$ \\ 1) Department of Electronic and Electrical Engineering, Pohang University of Science and Technology, San 31, Hyoja-dong, \\ Nam-gu, Pohang, Kyung-buk, 790-784, Republic of Korea. 2) Department of Information and Communication \\ Engineering, Dongguk University, Sukjang-dong, Kyungju, Kyung-buk, 780-714, Republic of Korea. \\ E-mail: swkim@postech.ac.kr
}

(Received on December 18, 2006; accepted on July 12, 2007)

\begin{abstract}
The conventional roll force automatic gauge control (RFAGC) adjusts the roll gap to reduce the delivery thickness variation in the hot strip finishing mills. In this process, the information about the roll force is used to control the roll gap indirectly because the strip gauge cannot be measured owing to insufficient space in the mill and the hostile environment. Moreover, there exists an uncertainty in thickness due to roll eccentricity. Therefore, a small gain is preferable in avoiding product defects when this uncertainty is taken into account in RFAGC. However, in such a case, partially uncontrolled variation still remains on delivery thickness because of insufficient control input. To overcome this drawback, a feed-forward controller (FFAGC) was developed. ${ }^{12)}$ This scheme adjusts the roll gap to reduce variations in the current stand by using an estimated uncontrolled delivery thickness variation of the previous stand. Although this FFAGC has an advantage over RFAGC, it still has a problem: The FFAGC cannot cope with the continuous variation of system parameters and circumstance because the existing feed-forward controller has been designed as a low pass filter with constant weights. In other words, desirable control performance in the automatic gauge control system cannot be obtained owing to a phase delay and filtering errors.

To overcome the problem, it is essential to estimate the long-term thickness variation exactly in the hard environment, to select a proper gain and to synchronize the feed-forward control input under a time varying speed of the gauge control process. In this paper, an enhanced adaptive filtering structure is proposed to improve the controller performance. And the relation between the gains of RFAGC and FFAGC are discussed to select a proper gain. The proposed controller can readily be implemented on the conventional control system, improving the performance of the system without additional cost. This improvement was demonstrated by using the real coil data which are collected under the proposed control scheme in POSCO.
\end{abstract}

KEY WORDS: automatic gauge control; feed forward control; adaptive signal processing.

\section{Introduction}

In the hot rolling process, the target of a multi-stand mill is to reduce the strip thickness $(h)$ while the strip passes through each stand by controlling the position of the hydraulic capsules $(S)$. However, the strip gauge cannot be measured because of the insufficient space in the mill and the hostile environment. The rolling force $(P)$ is the only measurement in the roll force automatic gauge controller. In this controller, the gain is usually adjusted to be less than that for a stable system. The smaller gain guarantees more stability in the large variation of the mill modulus. However, a small gain reduces performance as in the reduction ratio of entry thickness and delivery thickness variations. ${ }^{1-6)}$

To overcome the drawbacks mentioned above, a feed-forward scheme has been proposed. ${ }^{12)}$ It can reduce the uncontrolled variation under small gains of RFAGC. Specifically, this method extracts a long-term thickness variation signal from the delivery thickness variation of the previous stand and then uses this signal as an input in the current stand.
Consequently, the remaining delivery thickness variation can be reduced. It is very useful when the strips have a vivid skid mark. ${ }^{89}$ Here, this skid mark signal means a long-term thickness variation in the rolling process caused by local slab temperature fluctuation in a reheating furnace, and is the major part of the entry thickness variation. However, the conventional FFAGC could not cope with the variation of RFAGC gains, system parameters and circumstance.

This paper examines the influence of delivery thickness variation by analyzing two input variations as affected by RFAGC gains, namely, the entry thickness variation and the roll eccentricity variation. We explain the need for FFAGC by demonstrating that it is difficult to perfectly control the delivery thickness by adjusting the RFAGC gains alone. On the basis of this analysis, the relationship between FFAGC gains and RFAGC gains will also be examined.

To improve the performance of the existing FFAGC, this paper proposes an enhanced adaptive filtering method that extracts the long-term variation in the heavy noise environ- 
ment. This method contains several techniques such as an adaptive line enhancer which can adaptively extract a timevarying signal, an initial frequency estimator to enhance the convergence rate, and a linear phase FIR filter to reduce noises without distortion.

The rest of the paper is organized as follows. The structure of the hot strip finishing mill including RFAGC and FF AGC is introduced, and the stability related to gains is discussed in the next section. An adaptive filter structure to estimate the long-term thickness variation is proposed and implemented in Secs. 3 and 4. Finally, we introduce several experiments carried out in POSCO. Their results show the effectiveness of the proposed algorithm.

\section{Thickness Control in Hot Rolling Plant}

\subsection{Thickness Variation in Hot Strip Finishing Mill}

Seven mill stands are installed in the hot rolling process in POSCO. Each stand reduces the delivery thickness to the desired thickness, which is determined by the finishing mill setup unit (FSU) that decides all the setup values of the hot strip finishing mill. Generally, RFAGC and the monitor AGC are used to adjust the delivery thickness. Specifically, RFAGC controls the roll gap to maintain the roll force decided by FSU. The monitor AGC adjusts the roll gaps using $\mathrm{X}$-ray sensor data. The X-ray thickness sensor is installed only behind the final stand, therefore, the delivery thickness of the other stands cannot be measured. Therefore, this controller cannot work perfectly in the other stands because of time delays between the sensor and stands. Fig. 1 shows the layout of the structure of the hot strip rolling mill. ${ }^{1)}$
A delivery thickness signal usually consists of a low frequency component and several high frequency components. Fig. 2 shows the frequency analysis of a general delivery thickness. These components are the results from different causes such as roll eccentricity and entry thickness variation. Specifically, the low frequency component is related to a skid mark pattern which is determined by the gap of skids in a reheating chamber. And the period of roll eccentricity is determined by the speed of backup and working rolls, so it is shorter than that of skid mark. Therefore, it is related to high frequency components. Although those components are distinguished easily, their frequencies vary according to the circumstances including the ingredient ratio, thickness, roll speed, and temperature of a strip. In the next session, we discuss the relation between the delivery thickness and those components.

\subsection{Roll Force AGC}

The RFAGC uses force feedback to change the capsule position via the inverse of the mill modulus. However, the true roll gap cannot be exact because of the roll eccentricity and deformation, even if the position of roll can be measured exactly. Therefore, we can modify the basic equation of gauge control by adding the roll gap disturbance $(w)$ which presents the difference between the true roll gap and the measured value. ${ }^{10)}$ This value means the roll gap disturbance caused by roll eccentricity and measurement error on a position sensor of work roll. These linear equations describing the behavior of the stand and strip and considering the roll eccentricity effect are:

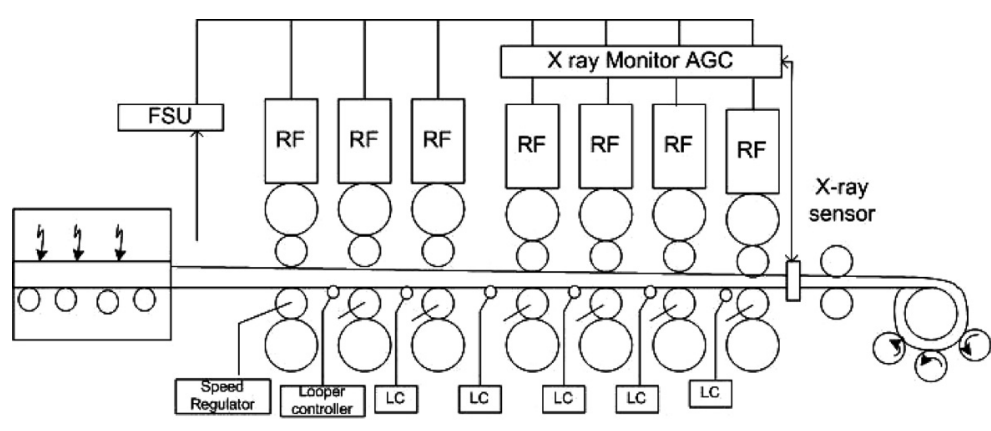

Stands

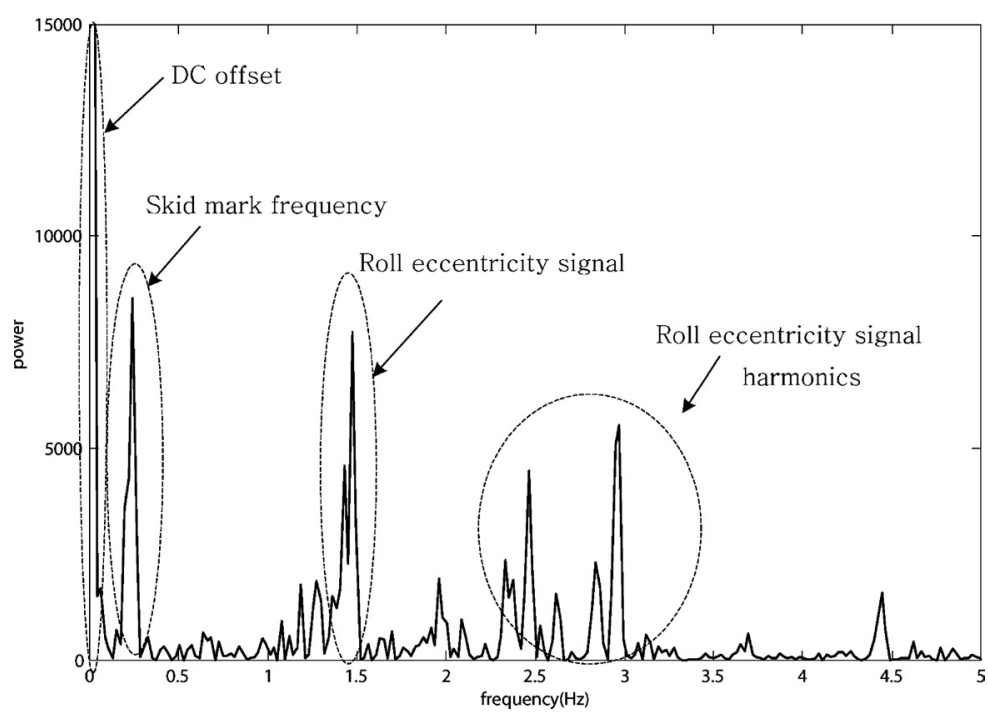

Fig. 1. The structure of hot rolling plant in POSCO.

Fig. 2. Frequency components of a delivery thickness variation. 


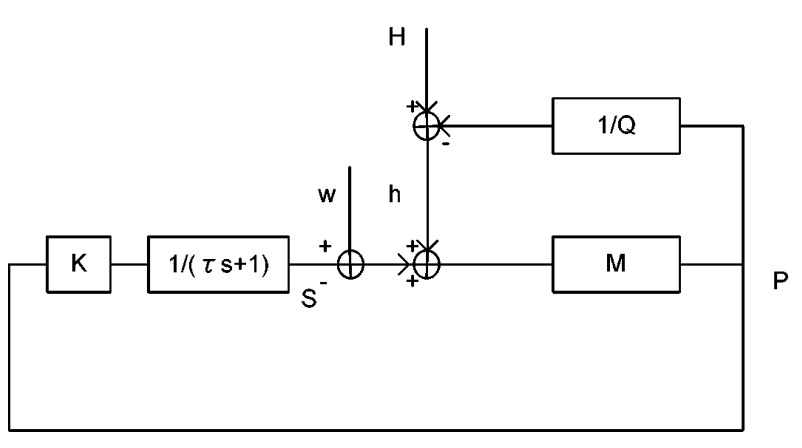

Fig. 3. Strip thickness control with roll eccentricity effect.

$$
\begin{gathered}
h=S_{\mathrm{c}}+\frac{P}{M}+w \\
P=Q(H-h) \ldots
\end{gathered}
$$

where $h$ is delivery thickness, $S$ is roll gap, $P$ is rolling force, $M$ is mill modulus, $Q$ is plastic coefficient, and $H$ means entry thickness. Figure 3 shows the thickness control loop that uses force $(P)$ feedback from a load cell to drive the hydraulic capsule position $S_{\text {c }}$. The gauge controller has the ideal gain $(K=-1 / M)$ since the steady state gain of the thickness sensitivity $\left(h=S_{\mathrm{h}} H\right)$ will be zero. For robust stability, the closed-loop poles should be analyzed for a change of $\pm 0.2 \bar{M}$ where $\bar{M}$ is the nominal mill modulus. The mill modulus changes because of the temperature and work roll wear while it can only be estimated when strip is not being rolled. If $K=-\alpha / \bar{M}$ the equation is:

$$
\begin{gathered}
h=\frac{Q\left(\tau_{s}+1-\frac{M}{\bar{M}} \alpha\right)}{M\left(\tau_{s}+1\right)+Q\left(\tau_{s}+1-\frac{M}{\bar{M}} \alpha\right)} \times H \\
+\frac{M\left(\tau_{s}+1\right)}{M\left(\tau_{s}+1\right)+Q\left(\tau_{s}+1-\frac{M}{\bar{M}} \alpha\right)} \times w . .
\end{gathered}
$$

For stability, the characteristic equation is ${ }^{10)}$ :

$$
s+\frac{1}{\tau} \frac{Q}{M+Q}\left[1+\frac{M}{Q}-\frac{M}{\bar{M}} \alpha\right]=0 .
$$

Since $\tau>0, M>0$ and $Q>0$ then the closed-loop system is stable if and only if:

$$
1+\frac{M}{Q}-\frac{M}{\bar{M}} \alpha>0 .
$$

or

$$
\frac{\alpha}{\bar{M}}<\frac{M+Q}{M Q}
$$

For closed-loop stability, the worst case $\alpha$ is solved by these equation:

$$
\frac{\alpha}{\bar{M}}<\frac{1.2 \bar{M}+Q}{1.2 \bar{M} Q} \rightarrow \alpha<\frac{1.2 \bar{M}+Q}{1.2 Q}
$$

Since $0<M / Q<1$ robust stability is achieved if:

$$
\alpha<\frac{1}{1.2}
$$

The nominal thickness performance with $K=-\alpha / M$ is:

$$
\begin{aligned}
& h=\frac{1-\alpha}{1-\alpha+\bar{M} Q^{-1}} H=T_{\mathrm{h}} H \\
& h=\frac{\bar{M} Q^{-1}}{1-\alpha+\bar{M} Q^{-1}} w=T_{\mathrm{w}} w
\end{aligned}
$$

These equations show that a proper $\alpha$ is required to provide the stability, and the two nominal performance (determined by $T_{\mathrm{h}}$ and $T_{\mathrm{w}}$ ) have a trade-off relation. In other words, a large $\alpha$ reduces the entry thickness variation but it does not reduce the effect of roll eccentricity well. On the other hand, a small gain can reduce the effect of roll eccentricity but cannot reduce the entry thickness variation. This problem should be solved to improve the performance. Therefore, a feed-forward control scheme has been used in the finishing mills. This scheme is working under the small $\alpha$ because it guarantees the stability of the system and reduces the effect of roll eccentricity.

\subsection{Trade-off Relation between Delivery Thickness variation and RFAGC Gain}

In the previous section, the relations among the RFAGC gain $\alpha$, the roll eccentricity effect, and the reduction ratio of variation were examined using the nominal equations. In this section, these relations are revealed by the field data. The thickness data of stand 6 were used since thin strips are easily affected by the roll eccentricity in general and have distinctive the long-term thickness variations. This paper compared two continuously rolled strips, which were rolled under different RFAGC gains although they had the same kind of steel and the same target thicknesses.

Figures $\mathbf{4}$ and $\mathbf{5}$ show delivery thickness variations with different RFAGC gains in stands 6 and 7, respectively. In Fig. 4, dashed line means long term variation signal filtered by conventional IIR filter. The upper part in Figs. 4 and 5 shows the cases in which small gains were used. They show that the effect of roll eccentricity does not remain but a large long-term thickness variation still remains. In contrast, the lower part in the figures represent the cases in which a large gain was used, and they show that a large effect of roll eccentricity remains, and this remains in effect until after the final thickness variation in stand 7.

\section{Adaptive Feed-forward Automatic Gauge Control}

\subsection{Feed-forward Automatic Gauge Control Scheme}

The FFAGC adjusts the roll gap in the current stand by estimating the long-term thickness variation of the previous stands. Therefore, it requires the relation between the roll gap of the current stand and the exit thickness of the previous stand. In the case of no disturbance, we can consider a modified equation similar to (1). Here, $S_{\mathrm{f}}$ is the roll gap control input by FFAGC and $\Delta H$ means the entry thickness variation. Here, the entry thickness variation is the same variable as the delivery thickness variation in the previous stand. 

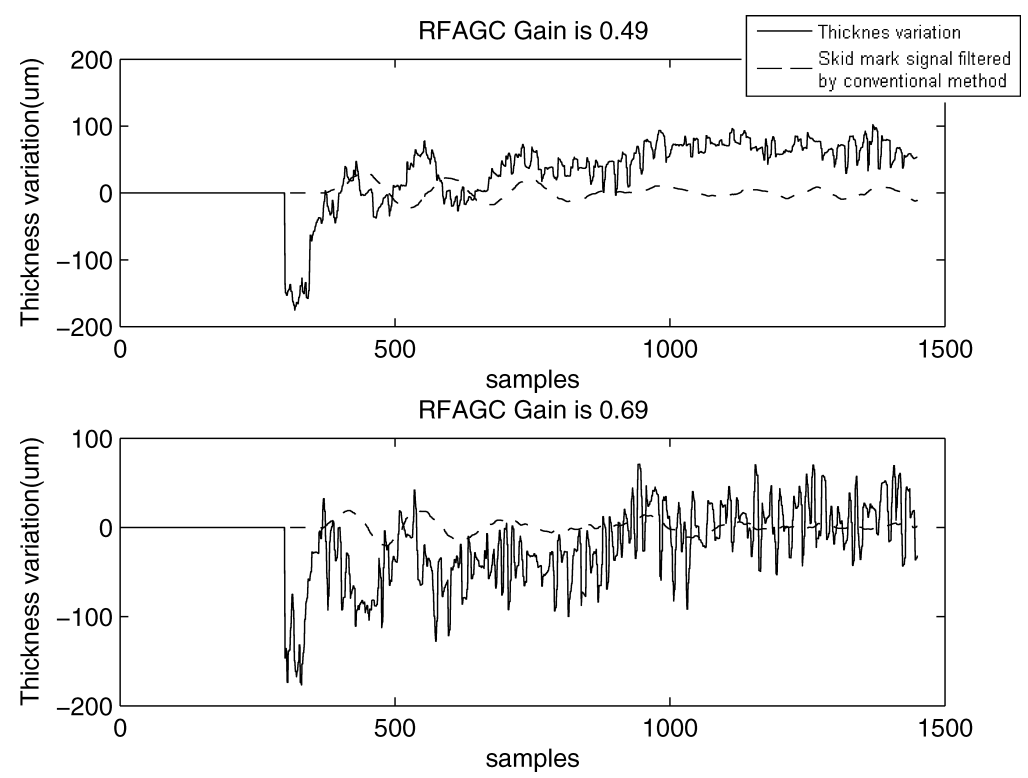

Fig. 4. Thickness variation with different gain in stand 6 .
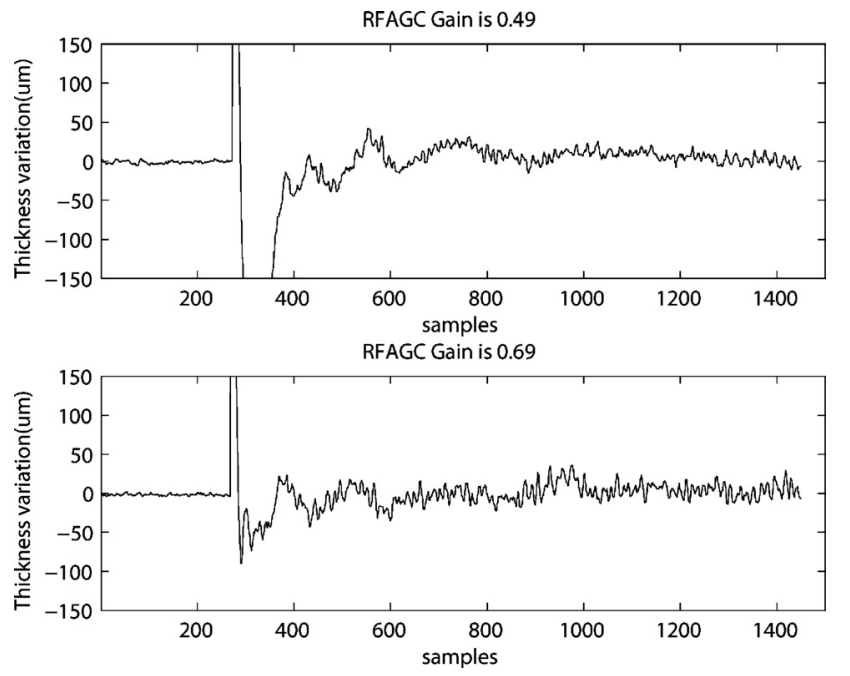

Fig. 5. Thickness variation with different gain in stand 7 .

$$
h=S_{\mathrm{c}}+\frac{P}{M}+S_{\mathrm{f}}
$$

As the previous derivation, we can get the following nominal equation easily.

$$
\Delta h=\frac{1-\alpha}{1-\alpha+\bar{M} Q^{-1}} \Delta H+\frac{\bar{M} Q^{-1}}{1-\alpha+\bar{M} Q^{-1}} \Delta S_{\mathrm{f}}
$$

Assuming $\Delta h=0$, the following equation related to the desired additional roll gap set-point is obtained.

$$
\Delta S_{\mathrm{f}}=-(1-\alpha) \frac{Q}{\bar{M}} \Delta H
$$

This equation means that the uncontrolled variation can be reduced with additional roll gap control (FFAGC), and that the gain is related to the gain of RFAGC. Figure 6 shows the structure of FFAGC. In the real implementation, an estimated $\Delta h$ in the previous stand is stacked in memory, filtered, delivered and finally used instead of the exact $\Delta H$ in the current stand.

\subsection{Enhanced Adaptive Filter Structure Design}

In the FFAGC, it is important to design the adaptive filter extracting long term thickness variation exactly. In this session, we derived an equation for estimating the initial frequency for fast convergence and we proposed a hybrid filter structure mixed with a linear phase FIR filter and a modified adaptive line enhancer. Additionally, an adaptive step size control algorithm is suggested for fast convergence.

\subsubsection{Linear Phase FIR filter}

A linear phase FIR filter can be used to reduce noises without phase distortion because of the distance between stands. In other words, we can make good use of the samples between the two stands before we reach the control time to estimate the long-term thickness variation. If the FIR filter is of a high order, it will work well with constant group delays in the long-term thickness variation frequency region. However, it takes about $0.6 \mathrm{~s}$ before reaching the next stand where sampling time is $10 \mathrm{~ms}$, which makes the tap of FIR filter limited to the 31 st order. Unfortunately, the frequency of the long-term thickness variation is so low that the proper FIR band-pass filter cannot be designed in this low frequency region. Only the FIR low pass filter is designed to reject noises and high harmonic frequencies. We adopted a design method of linear-phase FIR filters using an IDEF. This linear-phase FIR filter preserves the original waveform and rejects high frequency noises. ${ }^{4,5,7)}$

\subsubsection{Estimation algorithm for Initial Frequency}

As mentioned earlier in the paper, the skid mark signal is a thickness variation caused by the local slab temperature fluctuations in a heating furnace. Therefore, we can approximately estimate the frequency of the skid mark if the information about the slab in a heating furnace can be used. Figure 7 shows the changes in the skid mark frequency in rolling processes. Since the volume is not changed, the frequency is related to the skid gap in a heating furnace, the slab thickness, and the slab width. Therefore, the frequency of the skid mark can be roughly estimated using this information. The greater is the accuracy of estimated frequency, 

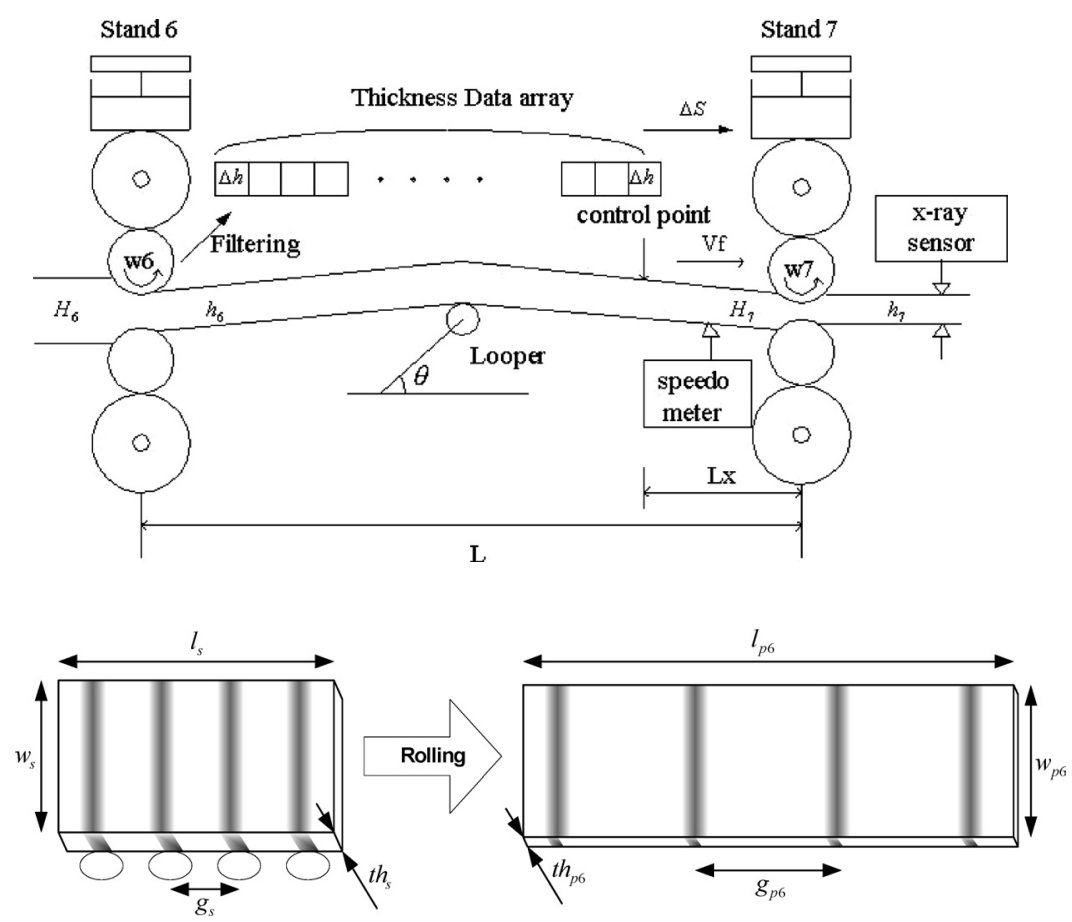

Fig. 6. The structure of feed forward AGC.

Fig. 7. Frequency changes of a skid mark in rolling process. the shorter is the convergence time. The following equations are used in estimating the initial frequency:

$$
\begin{gathered}
V_{\mathrm{o}}=l_{\mathrm{s}} \times w_{\mathrm{s}} \times t h_{\mathrm{s}}=l_{\mathrm{p}} \times w_{\mathrm{p}} \times t h_{\mathrm{p}} \ldots \ldots . . \\
l_{\mathrm{p}}=\frac{w_{\mathrm{s}} \times t h_{\mathrm{s}}}{w_{\mathrm{p}} \times t h_{\mathrm{p}}} l_{\mathrm{s}} \rightarrow g_{\mathrm{p}}=\frac{w_{\mathrm{s}} \times t h_{\mathrm{s}}}{w_{\mathrm{p}} \times t h_{\mathrm{p}}} g_{\mathrm{s}} \\
f_{\text {skid }}=\frac{v_{\text {roll }} / 60_{[\mathrm{mps}]}}{g_{p[\mathrm{~m}]}}=\frac{v_{\text {roll }} t_{\mathrm{p}}}{60 t_{\mathrm{s}} g_{\mathrm{s}}} \ldots \ldots
\end{gathered}
$$

where $V_{\mathrm{o}}$ is the volume of the slab, $l_{\mathrm{s}}$ and $l_{\mathrm{p}}$ are the lengths, $w_{\mathrm{s}}$ and $w_{\mathrm{p}}$ are the width, $t_{\mathrm{s}}$ and $t_{\mathrm{p}}$ are the thickness of a slab and a plate, respectively, $v_{\text {roll }}$ is the roll speed, $f_{\text {skid }}$ is the frequency of the skid mark, and $g_{\mathrm{s}}$ is the gap between the skid mark in a slab.

\subsubsection{Modified Adaptive Line Enhancer}

A general adaptive line enhancer (ALE) was used to enhance the sinusoids corrupted by noise, and to estimate and track the unknown frequencies of the sinusoids. We used a notch filter type ALE to estimate the long-term thickness variation. Some of the advantages of notch filtering are the easy control of bandwidth, the theoretically infinite null, and the capability of tracking sinusoidal frequencies, which can be retrieved or eliminated later. ${ }^{5)}$ Digital notch filtering has been used with various adaptive algorithms, for example, the notch filter using Infinite Impulse Response Band Pass Filter (IIR BPF).

$$
H(z)=\frac{(1-s)\left(w-z^{-1}\right)}{1-(1+s) w z^{-1}+s z^{-2}}
$$

Here, $s$ controls the bandwidth of this filter, $w$ decides a center frequency. For controlling the bandwidth variable, the following optimization algorithm was derived.

If the performance function is the power of error as $\xi_{\mathrm{e}}(s, w) \equiv E\left\{e^{2}{ }_{k}\right\}$, the following equation is derived.

$$
\xi_{e}(s, w)=\frac{a^{2}}{2}\left|1-e^{-j \theta_{0}} H\left(e^{j \theta_{0}}\right)\right|^{2}+\frac{2 \sigma_{\mathrm{v}}^{2}}{1+s}
$$

To minimize this performance function, $w$ is adjusted. Equation (19) is output power equation. This is used for construction the new performance function $\xi_{\mathrm{s}}(s, w)=$ $E\left\{y_{s, k}^{2}\right\}$.

$$
\begin{array}{r}
G(z)=\sqrt{\frac{1+s}{1-s}} H(z) \ldots \ldots \ldots \ldots \ldots \ldots \ldots \\
\xi_{s}(s, w)=\frac{a^{2}}{2} \cdot \frac{1+s}{1-s} \cdot\left|H\left(e^{j \theta_{0}}\right)\right|^{2}+\sigma_{\mathrm{v}}^{2}
\end{array}
$$

To minimize this performance function, $s$ is adjusted. To minimize the optimal cost function, the gradient-based algorithms were used in IIR adaptive Line enhancement filtering applications.

\subsubsection{Rejection of the Offset and Adaptive Step Size}

In the filtering problem of the long-term thickness variation, an input signal is the delivery thickness of the previous stand. This signal has many frequency components which need to reject the offset that degenerate the exact estimation of the long-term thickness variation. In this work, we used the IIR type low pass filter to reduce the effect of the offset in the modified ALE algorithm. Figure 8 shows the proposed structure of ALE algorithm. Table 1 explained the detail modified ALE algorithm. There are six update equations. The second equation is main adaptive line enhance algorithm. This equation requires two adaptive variables: $s_{k}$ and $w_{k}\left(\cos \theta_{k}\right)$. The fifth and sixth parts shows those update equations. Consequently, the output of ALE becomes the clean feed forward control input without offset and noises.

As for the variable step size $\left(\mu_{\theta}\right)$, when the step size becomes bigger, the convergence time of the algorithm gets 


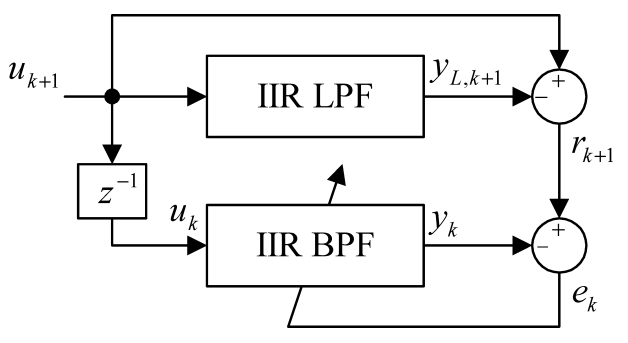

Fig. 8. The proposed structure of adaptive line enhancer.

Table 1. Modified ALE algorithm.

$:$ Variable for center frequency
$w_{k}=\cos \theta_{k}$
$:$ Main adaptive IIR BPF (ALE)
$y_{k}=\left(1+s_{k}\right) w_{k} y_{k-1}-s_{k} y_{k-2}+\left(1-s_{k}\right)\left(w_{k} u_{k}-u_{k-1}\right)$
$:$ IIR LPF for rejecting offset
$y_{L, k+1}=\alpha y_{L, k}+(1-\alpha) u_{k+1}$
$:$ Calculate errors
$r_{k+1}=u_{k+1}-y_{L, k+1}$
$e_{k}=r_{k+1}-y_{k+1}$
$:$ Update equation for Center frequency
$\alpha_{k}=\left(1+s_{k}\right) w_{k} \alpha_{k-1}-s_{k} \alpha_{k-2}-\sin \theta_{k}\left[\left(1+s_{k}\right) y_{k-1}+\left(1-s_{k}\right) u_{k}\right]$
$\mu_{\theta}=\mathrm{c} \cdot v_{s}$
$\theta_{k+1}=\theta_{k}+\mu_{\theta} \frac{e_{k} \alpha_{k}}{1+e_{k} \alpha_{k}}$
$:$ Update equation for bandwidth
$\beta_{k}=\left(1+s_{k}\right) w_{k} \beta_{k-1}-s_{k} \beta_{k-2}-\left(w_{k} e_{k-1}-e_{k-2}\right)$
$s_{k+1}=s_{k}+\mu_{s}\left[\frac{1}{\left(1-s_{k}\right)^{2}} y_{k}^{2}+\frac{1+s_{k}}{1-s_{k}} y_{k} \beta_{k}\right]$

shorter. In general, the faster is the roll speed $\left(v_{\mathrm{s}}\right)$, the higher is the frequency of a long-term thickness variation. Therefore, the speed information of a strip can be used to determine the adaptive step size. In the real implementation, the step size was given a larger value in the roll speed changing region because the center frequency of a longterm thickness variation changed quickly. Figure 9 shows the general pattern of roll speed in the hot strip finishing rolling in POSCO. The step size of region A is set to be bigger than that of the other regions in our adaptive filter.

\section{Implementation of FFAGC}

The stability of control system is not serious problem if the phase difference between a control signal and an entry thickness variation is not over than 90 degree. The longterm thickness variation in slab has very low frequency so it not difficult to keep this condition. To improve the performance of synchronization in the feed forward AGC, it is very important to exactly track the filtering signal and to get the exact control time delay in the following stand.

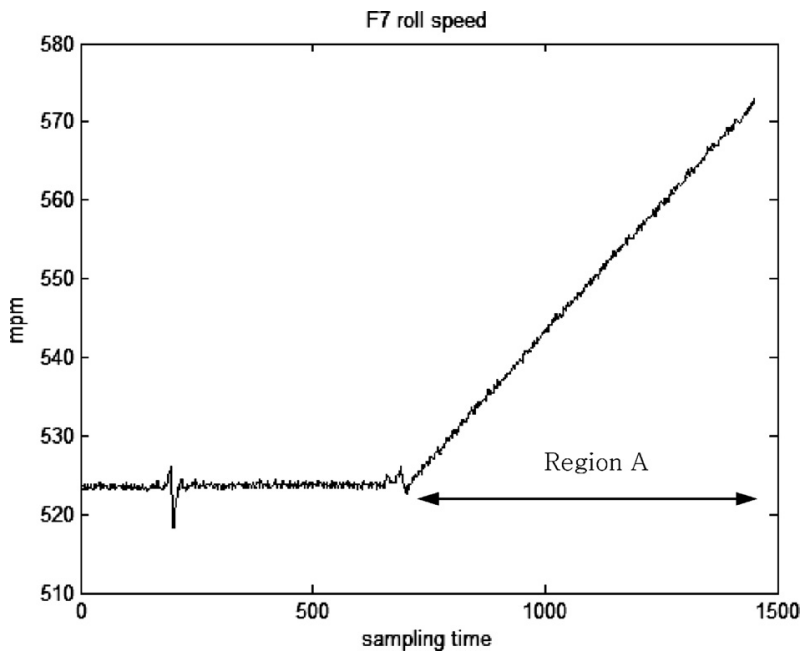

Fig. 9. The example of roll speed pattern.

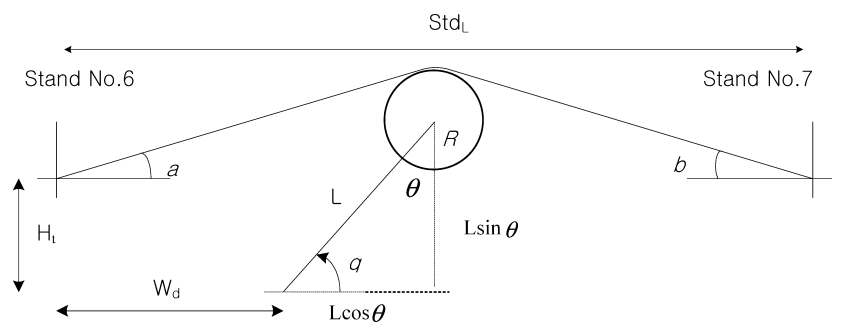

Fig. 10. The structure of looper.

\subsection{Calculation Strip Length between Stands}

In this section, the delay of the actuator and the changes in the strip length between two stands are investigated to estimate the exact control time delay. It is important that the strip length must be calculated exactly for strip tracking which depends on the strip tension and the looper angle in adjacent stands. The looper is an actuator that maintains the tension of a strip. As the strip length between stands becomes longer and the tension becomes lower, the looper angle grows higher to adjust the tension. Therefore, we use the looper angle to calculate the strip length exactly. Figure 10 shows the looper structure. The strip length between two stands is computed by the following equations:

$$
\begin{aligned}
& L_{\text {left }}=\sqrt{\left(L \sin \theta+R+H_{\mathrm{t}}\right)^{2}+\left(W_{\mathrm{d}}+L \cos \theta\right)^{2}} \\
& L_{\text {right }}=\sqrt{\left(L \sin \theta+R+H_{\mathrm{t}}\right)^{2}+\left(S t d_{\mathrm{L}}-W_{\mathrm{d}}+L \cos \theta\right)^{2}} \\
& L_{\text {strip }}=L_{\text {left }}+L_{\text {right }}
\end{aligned}
$$

where $L$ is a looper arm length, $R$ is a roller radius, $s t d_{\mathrm{L}}$ is a distance between stands, $H_{t}$ and $W_{d}$ are $y-x$ looper position, and $L_{\text {strip }}$ is the strip length between two stands.

\subsection{Gain Tuning of FFAGC}

From (13), it is revealed that the gain of FFAGC is related to that of RFAGC. Sometimes, the gain of RFAGC is changed by an expert or by the online gain tuning algorithm. In that case, the gain of FFAGC should be adjusted so that it will not degenerate the performance. Therefore, it is automatically adjusted to less than $(1-\alpha)$ in this paper. 


\section{Performance}

In this section, the performance of the modified ALE is compared with the conventional method, and the performance improvement of the adaptive FFAGC is presented using the real plant data.

\subsection{Performance of Proposed Adaptive Filter}

Figures 11 and 12 show a good performance of the proposed adaptive filter structure. To explain the filtering performance, the respective filtering results are compared with the off-line non-causal FIR of the 321st which represented as solid line. As shown in Fig. 11, a general IIR type low pass filter is used to extract the long-term thickness variation from the thickness variation signal. As mentioned before, this filtering result brings phase distortion because of no adaptation. The phase distortion is conspicuous in the rear part. On the other hand, the adaptive filter works very

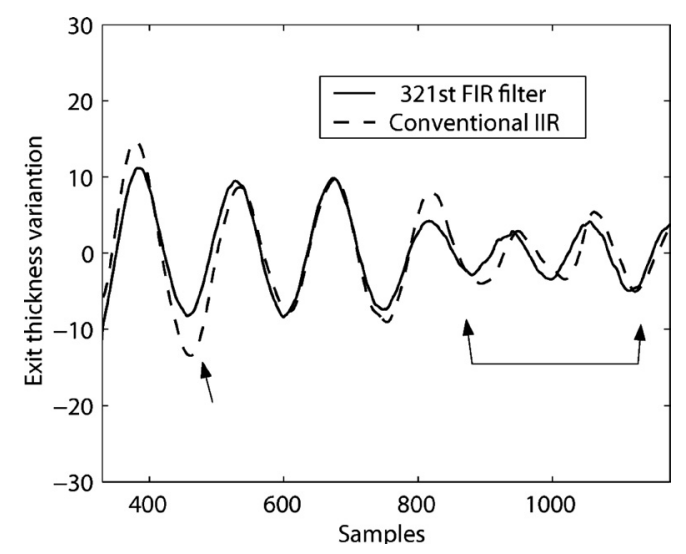

Fig. 11. The performance of general low pass filter.

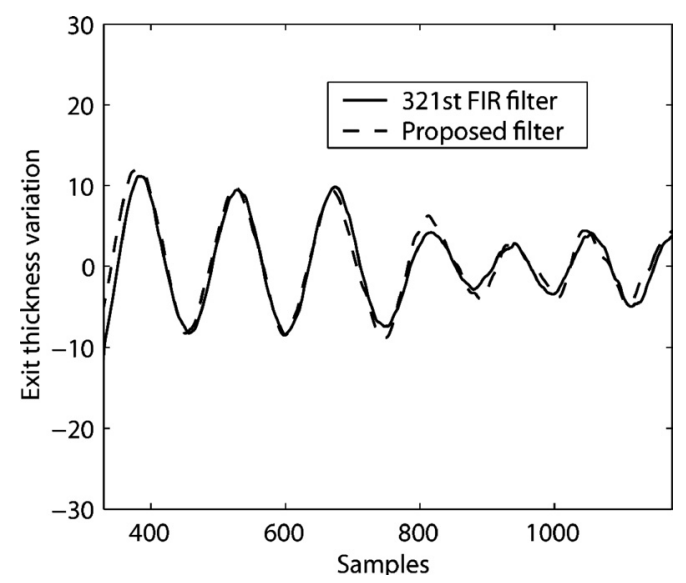

Fig. 12. The performance of the proposed adaptive filter. well with no sign of phase distortion as shown in Fig. 12. This difference is important since continuous variation of system parameters and circumstance. Figure 13 shows that the proposed filter works well in extracting the long-term thickness variation signal of a real plant.

\subsection{Experiment Results in the Hot Rolling Plant}

The proposed FF AGC is applied to stands 5 and 7 respectively because of the interference from the adjacent stands. To compare the performance, the proposed algorithm was implemented and tested in the second hot rolling plant, POSCO and the thickness variation was recorded. Specifically, this algorithm was applied to the middle gauge slabs because the long-term thickness variation was vivid on those slabs.

Figure 14 shows the final delivery thickness of strips under the conventional control, where the FFAGC was not applied to any stands. The long-term thickness variation appears notably and thickness variation is very large. Figure 15 shows the final delivery thickness of strips, where the proposed FFAGC is applied in the 5th stand only. The variation was less than that of the conventional control. However, the final thickness variation still remains.

Figure 16 shows the final delivery thickness of strips under the final proposed controller, where the proposed FFAGC was applied to the 5th and 7th stand respectively. As a result, the final thickness variations were considerably reduced in the proposed FFAGC compared with that of the conventional AGC. The proposed FFAGC significantly improved the quality of the rolled product without additional cost. This control scheme requires the only modification on

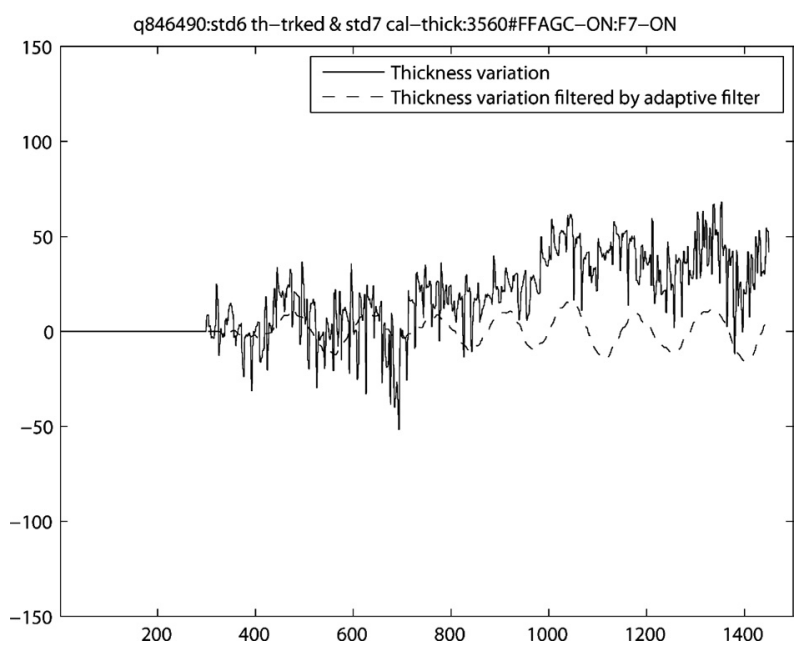

Fig. 13. Thickness variation and the filtered long-term thickness variation.

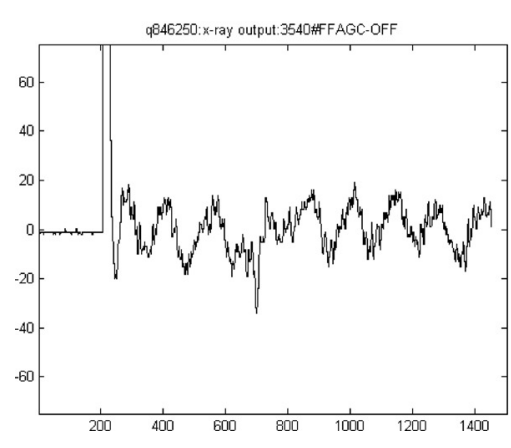

(C) 2007 ISIJ

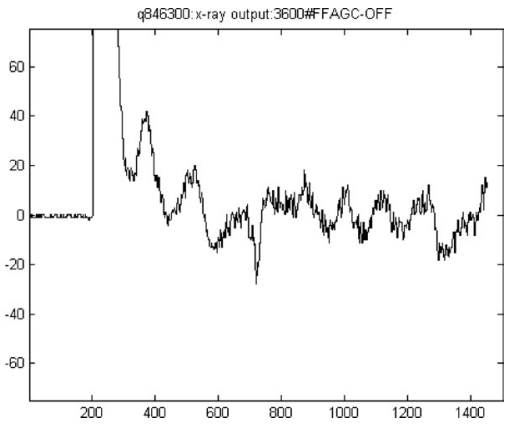

Fig. 14. Thickness variation under the conventional control (without FFAGC). 

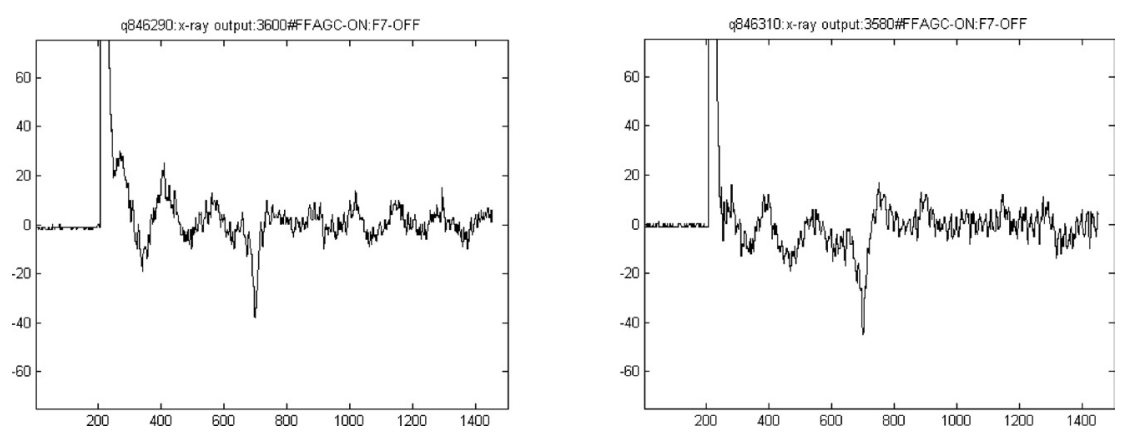

Fig. 15. Thickness variation under the proposed control (with FFAGC in only F5).
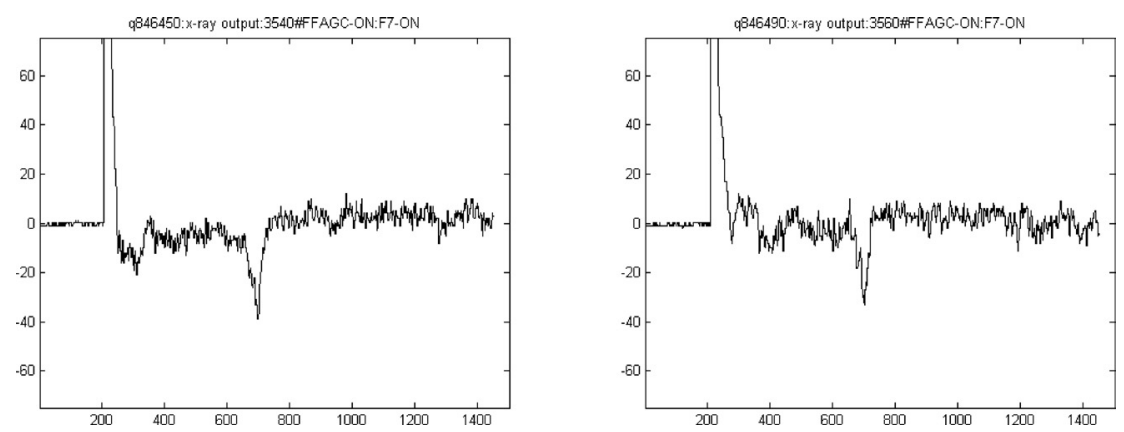

Fig. 16. Thickness variation under the proposed control (with FFAGC in F5 and F7).

the controller algorithm without additional sensors and system replacement. The proposed FFAGC was implemented and installed in the second hot rolling plant at POSCO.

\section{Conclusion}

The roll force controller in the conventional system was unable to reduce the variations perfectly in the delivery thickness because the gain was adjusted to keep the stability of the system. To overcome the drawback of the conventional control system, the FFAGC that was developed. However, the performance of the conventional FFAGC was degenerated since it could not cope with the continuous variations of system parameters and circumstances during the rolling process.

This paper found that the long-term thickness variation and roll eccentricity were the two major factors that caused the strip thickness variation, and a frequency domain analysis revealed that these components had different frequency bands. We also examined the relation between the gains and the thickness variation through the nominal equation and field data. The results showed that the FFAGC was more effective when the low frequency component was vivid under a low gain of the RFAGC. And this paper revealed that the proper gain of the FFAGC was related to the gain of the RFAGC.

To improve the performance of the conventional FFAGC, we developed an enhanced hybrid filter structure to extract the long-term thickness variation exactly and adjusted the gain of FFAGC automatically. This structure consisted of a linear phase FIR filter and the adaptive line enhancer. Additionally, the adaptive filter structure included an initial frequency estimator that used a skid gap and adaptive step size algorithm for fast adaptation. For an effective tracking performance, the angle of the looper and the speed of a strip were measured and used to estimate the exact control time delay. Our experiments demonstrated the effectiveness of the enhanced adaptive filter structure, and the performance of the proposed adaptive FFAGC was illustrated by the real processing data in POSCO.

\section{Acknowledgement}

This research was supported by the MIC (Ministry of Information and Communication), Korea, under the ITRC (Information Technology Research Center) support program supervised by the IITA (Institute of Information Technology Advancement) IITA-2006-C1090-0602-0013.

\section{REFERENCES}

1) V. B. Ginzburg: High-Quality Steel Rolling, Theory and Practice, Marcel Dekker, (1993), 175.

2) G. W. Rigler, H. R. Aberl, W. Staufer, K. Aistleitner and K. H. Weinberger: IEEE Trans. Ind. Appl., 32 (1986), No. 3, 599.

3) G. Keintzel, M. Schoisswohl, P. Vorstandlechner, K. Aistleitner and R. Schneeweis: IEEE Industrial Application Conf. Vol. 3, IEEE Industry Applications Society, (1998), 2263.

4) C. B. Rorabaugh: Digital Filter Designer's Handbook, McGraw-Hill Inc., New York, (1993), 271.

5) S. Salous: IEEE Trans. Educ., 41 (1998), No. 3, 229.

6) J. S. Cho, S. C. Hong and S. K. Choi: KACC 2000, ICROS, Korea, (2000), 1123.

7) B. Farhang-Boroujeny: IEEE Trans. Signal Process., 45 (1997), No. 2, 477.

8) Y. K. Lee, S. W. Kim and S. C. Hong: IECON '03. The 29th Annual Conf. of the IEEE, Vol. 3, IEEE Industrial Electronics Society, (2003), 2233.

9) Z. Wang, Y. Sun and K. Peng: Proc. American Control Conf., Vol. 6, Institute of Electrical and Electronics Engineers, Boston, (2005), 4242 .

10) G. Hearns and M. J. Grimble: ISIJ Int., 40 (2000), No. 10, 995.

11) Y. K. Lee, Y. J. Jang and S. W. Kim: IEICE Trans., E90-A (2007), No. 6.

12) Y. Washikita, M. Okamoto, T. Ooi and Y. Mori: ISIJ Int., 38, (1998), No. 9, 977. 